\title{
A MULTI-SPRING MODEL FOR BUCKLING ANALYSIS OF CRACKED TIMOSHENKO NANOBEAMS BASED ON MODIFIED COUPLE STRESS THEORY
}

\author{
Majid Akbarzadeh Khorshidi, Mahmoud Shariati \\ Ferdowsi University of Mashhad, Department of Mechanical Engineering, Mashhad, Iran \\ e-mail:mshariati44@um.ac.ir
}

\begin{abstract}
This paper develops a cracked nanobeam model and presents buckling analysis of this developed model based on a modified couple stress theory. The Timoshenko beam theory and simply supported boundary conditions are considered. This nonclassical model contains a material length scale parameter and can interpret the size effect. The cracked nanobeam is modeled as two segments connected by two equivalent springs (longitudinal and rotational). This model promotes discontinuity in rotation of the beam and additionally considers discontinuity in longitudinal displacement due to presence of the crack. Therefore, this multi-spring model can consider coupled effects between the axial force and bending moment at the cracked section. The generalized differential quadrature (GDQ) method is employed to discretize the governing differential equations, boundary and continuity conditions. The influences of crack location, crack severity, material length scale parameter and flexibility constants of the presented spring model on the critical buckling load are studied.
\end{abstract}

Keywords: buckling, crack, modified couple stress theory, Timoshenko nanobeam, spring model

\section{Introduction}

Many applicable structures have been used in micro- and nano-scale dimensions. Size effects are significant in the mechanical behavior of these structures. Since the classical continuum mechanics cannot predict the size effect, some size-dependent continuum theories have been developed to capture the size effect using some material length scale parameters. These parameters are related to inherent properties of materials, which become considerable in small scale structures. In view of the difficulties in determining these internal parameters, non-classical continuum theories involving only one material length scale parameter are desirable. A modified couple stress theory has been proposed by Yang et al. (2012). This theory uses only one material length scale parameter to capture the size-dependent behavior of structures and employs only the symmetric part of the couple stress tensor as a suitable measure of the continuum micro-rotation. Many studies have been done on the static and dynamic behavior of nanostructures based on the modified couple stress theory. Here we focuse on the static and dynamic behavior of the micro/nanobeams specially on the buckling of the nanobeams.

Park and Gao $(2006,2008)$ formulated a modified couple stress based model for the Euler-Bernoulli beam and that model was extended by Ma et al. (2008) for the Timoshenko beam using Hamilton's principle. Also, a microstructure dependent non-classical Reddy-Levinson beam model was developed by Ma et al. (2010), and the difference between the results obtained from the non-classical and the classical models was illustrated for static bending and free vibration. Simsek (2010) proposed analytical and numerical solution procedures for vibration of an embedded microbeam under action of a moving microparticle and studied the influences of the material length scale parameter, Poisson's ratio, velocity of the microparticle and the elastic 
medium constant on the maximum dynamic deflections of the microbeam. A size-dependent nonlinear Euler-Bernoulli beam model was presented by Xia et al. (2010). They studied nonlinear size-dependent static bending, postbuckling and the free vibration of beams, and Asghari et al. (2010) developed their model for Timoshenko beam theory. Ke and Wang (2011) investigated dynamic stability of FGM Timoshenko microbeams and showed the significance of the size effect on dynamic stability. Buckling analysis of microbeams with higher order theories and general boundary conditions was investigated by Mohammad-Abadi and Daneshmehr (2014). They revealed accuracy of the GDQ method for that modified couple stress based size-dependent buckling problem. Also, Mohammad-Abadi and Daneshmehr (2015) developed vibration analysis of composite laminated beams in order of microns using the GDQ method. Akbarzadeh Khorshidi and Shariati (2016b) presented a comprehensive solution for free vibration of a shear deformable S-FGM nanobeam by the GDQ method. Dehrouyeh-Semnani et al. (2015) investigated dynamic characteristics of axially moving Timoshenko microbeams using Hamilton's principle and Galerkin's method. An exact solution for prediction of postbuckling behavior of shear deformable nanobeams was presented by Akbarzadeh Khorshidi and Shariati (2015). Also, Akbarzadeh Khorshidi and Shariati (2016a) investigated the propagation of the stress wave in a shear deformable nanobeam and evaluated the effects of shear deformation, material length scale parameter and Poisson's ratio on the phase velocity of it.

It is well known that cracks increase flexibility of a structure. Therefore, the presence of a crack leads to reduction of the stiffness of a structure. So, a simple and accurate model should be used to determine this reduction in stiffness. For this reason, the cracked beam is modeled as two segments connected by means of massless springs (Freund and Herrmann, 1976; Adams et al., 1978). Rice and Levy (1972) viewed the plate as a strictly two dimensional continuum with a local reduction in bending and extensional stiffness along the crack line. This model, which has become known as the line spring model for surface flaws, was also discussed in detail by Rice (1972). Most of the studies which have been recently done utilized an equivalent massless rotational spring at the cracked section. Thus, at the cracked section, a discontinuity in rotation due to bending must be considered. This model has been extensively used for vibration, buckling and postbuckling analyses of cracked structures like cracked beams. Chaudhari and Maiti (2000) presented a method of modelling for transverse vibrations of geometrically segmented slender cracked beams. Lele and Maiti (2002) studied transverse vibration of short beams to detect the location of a crack. Yang and Chen (2008) investigated free vibration and elastic buckling of beams made of FGMs containing three open edge cracks. Ke et al. (2009) studied the postbuckling response of edge cracked FG Timoshenko beams. Also, there are many other research works in the context of cracked structures by using the equivalent spring model, see for instance, Chati et al. (1997), Krawczuk et al. (2003), El Bikiri et al. (2006), Kitipornchai et al. (2009), Yan et al. (2011). But, these models cannot satisfy the multiple discontinuities at the cracked section. Loya et al. (2006) considered a rotational and an extensional spring at the cracked section for bending vibration of a Timoshenko cracked beam. Thus, in addition to the discontinuity in rotation due to bending, a discontinuity in the transverse deflection due to shearing has been also defined at the cracked section. However, the contribution of the extensional spring to the strain energy of the system is small in comparison with that of the rotational spring, nevertheless, this discontinuity has been considered for coherency with the general derivation of compliance for cracked beams (Okamura et al., 1973; Tharp, 1987).

There are a few studies on cracked micro/nanostructures, which are mostly different types of vibration analyses of cracked nanobeams based on a nonclassical continuum theory by means of the rotational spring model. Flexural vibrations of cracked nanobeams based on the theory of nonlocal elasticity applied to Euler-Bernouilli beams was studied by Loya et al. (2009). They proposed a model containing a rotational and a longitudinal elastic spring at the cracked section and defined compatibility relations of this model. This model presents a discontinuity in the 
rotation and a discontinuity in the longitudinal displacement. But, they simplified their model to one spring only (rotational spring) by some assumptions, and many authors followed them for free transverse vibration analyses of cracked nanobeams (Hasheminejad et al., 2011; Torabi and Nafar Dastgerdi, 2012; Hosseini-Hashemi et al., 2014). Of course, those assumptions can be acceptable for free transverse vibration. Also, Hsu et al. (2011) investigated longitudinal frequency of a cracked nanobeam by means of the longitudinal spring model. They expressed continuity conditions as a relation between the longitudinal displacement and axial force, and nelegted the discontinuity in rotation. Also, Loya et al. (2014) analysed torsional vibrations of a nanorod with a circumferential crack using a nonlocal elasticity model. They utilized a torsional elastic spring at the cracked section to consider additional strain energy due to the presence of the crack, so, a discontinuity in the torsion angle of the rod at the cracked section is introduced. Therefore, an appropriate model can be selected to simulate the influence of the crack based on the type of analysis and the applied loadings.

This paper makes one of the first attempts to investigate the buckling of cracked nanobeams based on a modified couple stress theory. This modified couple stress based Timoshenko beam model contains a material length scale parameter and can interpret the size effect. The present model considers coupled effects between the axial force and bending moment using a longitudinal and a rotational spring at the cracked section. Govrning equations are derived by using the principle of minimum potential energy. The Generalized Differential Quadrature (GDQ) method is employed to solve the governing differential equations. Then the critical buckling loads for different crack locations, crack severities and ratios of the material length scale parameter to thickness are computed, and the obtained results are compared with those corresponding to the classical beam model.

\section{Formulation}

\section{1. $\quad$ Modified couple stress theory}

In this Section, a modified couple stress theory (Yang et al., 2012) with only one material length scale parameter is employed to capture the size effect. According to this theory, the strain energy $U$ in an isotropic linear elastic beam under an axial compressive load $P$ at both ends is defined as (Mohammad-Abadi and Daneshmehr, 2014)

$$
U=\frac{1}{2} \int_{\Omega}\left(\sigma_{i j} \delta \varepsilon_{i j}+m_{i j} \delta \chi_{i j}\right) d v-\frac{1}{2} \int_{0}^{L} P\left(\frac{\partial w}{\partial x}\right)^{2} d x \quad i, j=1,2,3
$$

where $\varepsilon, \sigma, \chi$ and $\mathbf{m}$ are the strain tensor, Cauchy stress tensor, symmetric curvature tensor and the deviatoric part of the couple stress tensor, respectively. These tensors are defined as

$$
\begin{array}{rlrl}
\varepsilon_{i j} & =\frac{1}{2}\left(\nabla \mathbf{u}+(\nabla \mathbf{u})^{\mathrm{T}}\right)=\frac{1}{2}\left(u_{i, j}+u_{j, i}\right) & \sigma_{i j} & =\lambda \operatorname{tr}\left(\varepsilon_{i i}\right) \delta_{i j}+2 \mu \varepsilon_{i j} \\
\chi_{i j}=\frac{1}{2}\left(\nabla \theta+(\nabla \theta)^{\mathrm{T}}\right)=\frac{1}{2}\left(\theta_{i, j}+\theta_{j, i}\right) & m_{i j}=2 \ell^{2} \mu \chi_{i j}
\end{array}
$$

where $\ell$ is the material length scale parameter which is mathematically the square root of the ratio of the modulus of curvature to the modulus of shear and is physically a property measuring the effect of couple stress (Park and Gao, 2006; Ma et al., 2008). This parameter can be determined from torsion tests of slim cylinders of different diameters or bending tests of thin beams of different thickness. Also, $\lambda$ and $\mu$ are Lame's constants and are defined as

$$
\lambda=\frac{\nu E}{(1+\nu)(1-2 \nu)} \quad \mu=\frac{E}{2(1+\nu)}
$$


where $E$ is Young's modulus and $\nu$ is Poisson's ratio. The rotation vector $\boldsymbol{\theta}$ is given as

$$
\theta=\frac{1}{2} \operatorname{curl}(\mathbf{u})
$$

\subsection{Displacement field}

Consider a beam of length $L$, width $b$ and thickness $h$. The Timoshenko beam theory is employed to describe the effect of shear deformation. According to the rectangular Cartesian coordinate system shown in Fig. 1 , where the $x$-axis is coincident with the centroidal axis of the undeformed beam, the $y$-axis is the neutral axis and the $z$-axis is the symmetry axis, the displacement field in a Timoshenko beam is described as

$$
u_{1}=u(x, t)-z \varphi(x, t) \quad u_{2}=0 \quad u_{3}=w(x, t)
$$

where $u_{1}, u_{2}$ and $u_{3}$ are, respectively, the $x-, y$ - and $z$-components of the displacement vector $\mathbf{u}$, $u$ and $w$ are, respectively, the $x$ - and $z$-components of the displacement vector of a point located on the beam axis, and $\varphi$ is the angle of rotation of the cross-section.

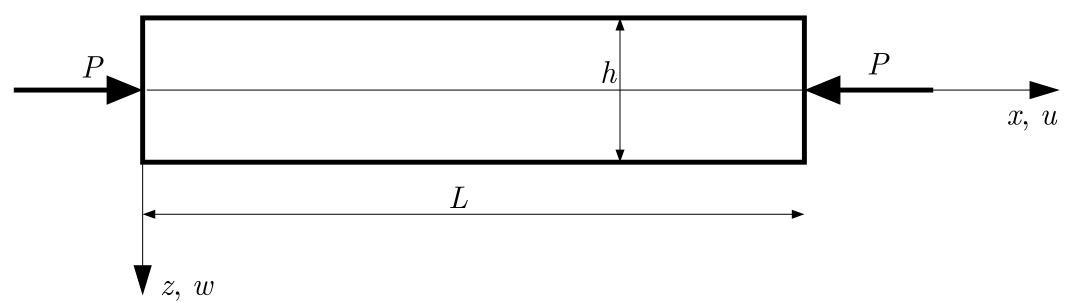

Fig. 1. Beam configuration and coordinate system

From Eqs. $(2.2)_{1}$ and (2.5), the nonzero strains are given as

$$
\varepsilon_{x x}=\frac{\partial u_{1}}{\partial x}=\frac{\partial u}{\partial x}-z \frac{\partial \varphi}{\partial x} \quad \varepsilon_{x z}=\frac{1}{2}\left(\frac{\partial u_{1}}{\partial z}+\frac{\partial w}{\partial x}\right)=\frac{1}{2}\left(\frac{\partial w}{\partial x}-\varphi\right)
$$

and from Eqs. $(2.2)_{2}$ and (2.5), the nonzero stresses are given as

$$
\begin{aligned}
\sigma_{x x} & =(\lambda+2 \mu) \varepsilon_{x x}=(\lambda+2 \mu)\left(\frac{\partial u}{\partial x}-z \frac{\partial \varphi}{\partial x}\right) \\
\sigma_{x z} & =2 \mu \varepsilon_{x z}=\mu\left(\frac{\partial w}{\partial x}-\varphi\right)
\end{aligned}
$$

Using Eqs. (2.4) and (2.5), the nonzero component of the rotation vector is obtained as

$$
\theta_{y}=-\frac{1}{2}\left(\varphi+\frac{\partial w}{\partial x}\right)
$$

and from Eqs. (2.2) $)_{3,4}$ and (2.8), we have

$$
\chi_{x y}=-\frac{1}{4}\left(\frac{\partial \varphi}{\partial x}+\frac{\partial^{2} w}{\partial x^{2}}\right) \quad m_{x y}=-\frac{1}{2} \ell^{2} \mu\left(\frac{\partial \varphi}{\partial x}+\frac{\partial^{2} w}{\partial x^{2}}\right)
$$

\subsection{Governing equations}

Substituting Eqs. (2.6), (2.7) and (2.9) into Eq. (2.1), the total strain energy of the Timoshenko nanobeam is achieved. Then, using the principle of minimum potential energy and 
fundamental lemma of the calculus of variation, the governing equations of the Timoshenko nanobeam in terms of displacements are obtained as

$$
\begin{aligned}
& (\lambda+2 \mu) A \frac{\partial^{2} u}{\partial x^{2}}=0 \\
& K_{s} \mu A\left(\frac{\partial^{2} w}{\partial x^{2}}-\frac{\partial \varphi}{\partial x}\right)-\frac{1}{4} \ell^{2} \mu A\left(\frac{\partial^{4} w}{\partial x^{4}}+\frac{\partial^{3} \varphi}{\partial x^{3}}\right)-P \frac{\partial^{2} w}{\partial x^{2}}=0 \\
& K_{s} \mu A\left(\frac{\partial w}{\partial x}-\varphi\right)+\frac{1}{4} \ell^{2} \mu A\left(\frac{\partial^{3} w}{\partial x^{3}}+\frac{\partial^{2} \varphi}{\partial x^{2}}\right)+(\lambda+2 \mu) I \frac{\partial^{2} \varphi}{\partial x^{2}}=0
\end{aligned}
$$

where $A$ and $I$ are, respectively, the beam cross-sectional area and the second moment of cross-sectional area. Also, $K_{s}$ is the Timoshenko shear coefficient which is introduced as a correction factor to account for the non-uniformity of the shear strain over the beam cross-section (Park and Gao, 2006) and is defined with Poisson's ratio as $K_{s}=(5+5 \nu) /(6+5 \nu)$.

Note that all of the body forces and body couples are neglected in this study. Therefore, the governing equations presented in Eqs. (2.10) are identical to those given by Mohammad-Abadi and Daneshmehr (2014).

the boundary conditions for a simply supported beam are stated as

$$
u=w=Y=M=0 \quad \text { at } \quad x=0, L
$$

where $Y$ is the couple moment, which is a resultant of the couple stress component mxy. Also, $M$ is the conventional bending moment. These moments are defined as

$$
Y=\int_{A} m_{x y} d A=\frac{1}{2} \ell^{2} \mu A\left(\frac{\partial^{2} w}{\partial x^{2}}+\frac{\partial \varphi}{\partial x}\right) \quad M=\int_{A} \sigma_{x x} z d A=(\lambda+2 \mu) I \frac{\partial \varphi}{\partial x}
$$

So, the boundary conditions defined in Eq. (2.11), can be writen as

$$
u=w=\frac{\partial \varphi}{\partial x}=\frac{\partial^{2} w}{\partial x^{2}}=0 \quad \text { at } \quad x=0, L
$$

\subsection{Cracked nanobeam model}

Consider a crack at a distance $L_{c}\left(e=L_{c} / L\right)$ at the left end of the beam (Fig. 2). It is assumed that the crack is perpendicular to the beam surface and always remains open. As shown in Fig. 2, the cracked beam is modeled as two segments connected by two massless elastic springs (longitudinal and rotational) (Loya et al., 2009). This modeling promotes flexibility at the cracked section and introduces discontinuity in rotation and discontinuity in longitudinal displacement of the beam, which are proportional to coupled effects of the axial force and bending moment. Therefore, the continuity conditions are developed for the Timoshenko nanobeam as $\left(\right.$ at $\left.x=L_{c}\right)$

$$
\begin{array}{lcrl}
w_{1}=w_{2} & N_{1}=N_{2} & M_{1}=M_{2} & \\
Q_{1}-P \varphi_{1}=Q_{2}-P \varphi_{2} & \varphi_{2}-\varphi_{1} \propto f(N, M) & u_{2}-u_{1} \propto g(N, M)
\end{array}
$$

where subscripts 1 and 2 refer to the left segment and right segment of the beam divided by the crack. Also, $N$ and $Q$ are, respectively, the axial force and the shear force, which are defined as

$$
N=\int_{A} \sigma_{x x} d A=(\lambda+2 \mu) A \frac{\partial u}{\partial x} \quad Q=\int_{A} \sigma_{x z} d A=K_{s} \mu A\left(\frac{\partial w}{\partial x}-\varphi\right)
$$


Using Eqs. (2.12) and (2.15), the continuity conditions can be written as (at $x=L_{c}$ )

$$
\begin{aligned}
& w_{1}=w_{2} \quad \frac{\partial u_{1}}{\partial x}=\frac{\partial u_{2}}{\partial x} \quad \frac{\partial \varphi_{1}}{\partial x}=\frac{\partial \varphi_{2}}{\partial x} \\
& K_{s} \mu A\left(\frac{\partial w_{1}}{\partial x}-\frac{\partial w_{2}}{\partial x}+\varphi_{2}-\varphi_{1}\right)=P\left(\varphi_{1}-\varphi_{2}\right) \\
& \varphi_{2}-\varphi_{1}=K_{M M} \frac{\partial \varphi}{\partial x}+K_{M N} \frac{\partial u}{\partial x} \quad u_{2}-u_{1}=K_{N N} \frac{\partial u}{\partial x}+K_{N M} \frac{\partial \varphi}{\partial x}
\end{aligned}
$$

where $K_{M M}, K_{M N}, K_{N M}$ and $K_{N N}$ are the flexibility constants and in nondimensional forms they are given as $k_{1}=K_{M M} / L, k_{2}=K_{M N} / L, k_{3}=K_{N N} / L, k_{4}=K_{N M} / L$.

Then, governing equations (2.10) must be applied for each segment $(r=1,2)$ of the cracked beam, so, we have

$$
\begin{aligned}
& (\lambda+2 \mu) A \frac{\partial^{2} u_{r}}{\partial x_{r}^{2}}=0 \\
& K_{s} \mu A\left(\frac{\partial^{2} w_{r}}{\partial x_{r}^{2}}-\frac{\partial \varphi_{r}}{\partial x_{r}}\right)-\frac{1}{4} \ell^{2} \mu A\left(\frac{\partial^{4} w_{r}}{\partial x_{r}^{4}}+\frac{\partial^{3} \varphi_{r}}{\partial x_{r}^{3}}\right)-P \frac{\partial^{2} w_{r}}{\partial x_{r}^{2}}=0 \\
& K_{s} \mu A\left(\frac{\partial w_{r}}{\partial x_{r}}-\varphi_{r}\right)+\frac{1}{4} \ell^{2} \mu A\left(\frac{\partial^{3} w_{r}}{\partial x_{r}^{3}}+\frac{\partial^{2} \varphi_{r}}{\partial x_{r}^{2}}\right)+(\lambda+2 \mu) I \frac{\partial^{2} \varphi_{r}}{\partial x_{r}^{2}}=0
\end{aligned}
$$

where $0 \leqslant x_{1} \leqslant L_{1}$ and $L_{1} \leqslant x_{2} \leqslant L$.

\section{Rotational spring}

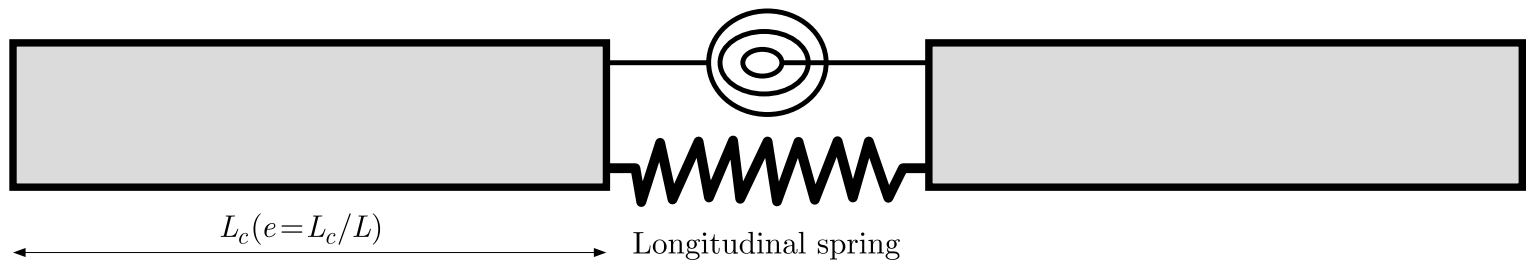

Fig. 2. Model of the cracked beam

\section{Solution procedure}

The GDQ method (Shu, 1991; Bellman et al., 1972; Shu and Du, 1997) is employed to discretize the governing equations, boundary conditions and continuity conditions, therefore, the differential equations are simplified to algebraic equations. In this method, the region is discretized into several sample points. The sample points are obtained as

$$
x_{i}=\frac{1}{2}\left(1-\cos \frac{(i-1) \pi}{N-1}\right) \quad i=1,2, \ldots, N
$$

According to the GDQ method (to find details, see Akbarzadeh Khorshidi and Shariati, 2016b; Shu and Du, 1997), governing equations (2.17) are discretized as

$$
(\lambda+2 \mu) A \sum_{j=1}^{N} c_{i j}^{(2)} u_{r}\left(x_{j}\right)=0
$$


and

$$
\begin{aligned}
& K_{s} \mu A\left(\sum_{j=1}^{N} c_{i j}^{(2)} w_{r}\left(x_{j}\right)-\sum_{j=1}^{N} c_{i j}^{(1)} \varphi_{r}\left(x_{j}\right)\right)-\frac{1}{4} \ell^{2} \mu A\left(\sum_{j=1}^{N} c_{i j}^{(4)} w_{r}\left(x_{j}\right)+\sum_{j=1}^{N} c_{i j}^{(3)} \varphi_{r}\left(x_{j}\right)\right) \\
& -P\left(\sum_{j=1}^{N} c_{i j}^{(2)} w_{r}\left(x_{j}\right)\right)=0 \\
& K_{s} \mu A\left(\sum_{j=1}^{N} c_{i j}^{(1)} w_{r}\left(x_{j}\right)-\varphi_{r}\left(x_{j}\right)\right)+\frac{1}{4} \ell^{2} \mu A\left(\sum_{j=1}^{N} c_{i j}^{(3)} w_{r}\left(x_{j}\right)+\sum_{j=1}^{N} c_{i j}^{(2)} \varphi_{r}\left(x_{j}\right)\right) \\
& +(\lambda+2 \mu) I\left(\sum_{j=1}^{N} c_{i j}^{(2)} \varphi_{r}\left(x_{j}\right)\right)=0
\end{aligned}
$$

where $i, j=1, \ldots, N$ and $c_{i j}^{(n)}$ is the weighting coefficient which can be found from the recursive formula (Akbarzadeh Khorshidi and Shariati, 2016b; Shu and Du, 1997).

The discretized boundary conditions are expressed as

$$
\begin{aligned}
& u_{1}\left(x_{1}\right)=w_{1}\left(x_{1}\right)=\sum_{j=1}^{N} c_{1 j}^{(1)} \varphi_{1}\left(x_{j}\right)=\sum_{j=1}^{N} c_{1 j}^{(2)} w_{1}\left(x_{j}\right)=0 \\
& u_{2}\left(x_{N}\right)=w_{2}\left(x_{N}\right)=\sum_{j=1}^{N} c_{N j}^{(1)} \varphi_{2}\left(x_{j}\right)=\sum_{j=1}^{N} c_{N j}^{(2)} w_{2}\left(x_{j}\right)=0
\end{aligned}
$$

also, continuity conditions (2.16) are discretized as

$$
\begin{aligned}
& w_{1}\left(x_{N}\right)=w_{2}\left(x_{1}\right) \quad \sum_{j=1}^{N} c_{N j}^{(1)} u_{1}\left(x_{j}\right)=\sum_{j=1}^{N} c_{1 j}^{(1)} u_{2}\left(x_{j}\right) \\
& \sum_{j=1}^{N} c_{N j}^{(1)} \varphi_{1}\left(x_{j}\right)=\sum_{j=1}^{N} c_{1 j}^{(1)} \varphi_{2}\left(x_{j}\right) \\
& K_{s} \mu A\left(\sum_{j=1}^{N} c_{N j}^{(1)} w_{1}\left(x_{j}\right)-\sum_{j=1}^{N} c_{1 j}^{(1)} w_{2}\left(x_{j}\right)+\varphi_{2}\left(x_{1}\right)-\varphi_{1}\left(x_{N}\right)\right)=P\left(\varphi_{1}\left(x_{N}\right)-\varphi_{2}\left(x_{1}\right)\right) \\
& \varphi_{2}\left(x_{1}\right)-\varphi_{1}\left(x_{N}\right)=K_{M M} \sum_{j=1}^{N} c_{N j}^{(1)} \varphi_{1}\left(x_{j}\right)+K_{M N} \sum_{j=1}^{N} c_{N j}^{(1)} u_{1}\left(x_{j}\right) \\
& u_{2}\left(x_{1}\right)-u_{1}\left(x_{N}\right)=K_{N M} \sum_{j=1}^{N} c_{N j}^{(1)} u_{1}\left(x_{j}\right)+K_{N N} \sum_{j=1}^{N} c_{N j}^{(1)} \varphi_{1}\left(x_{j}\right)
\end{aligned}
$$

Using discretized equations (3.2)-(3.5), a set of algebraic equations is established, and by solving the eigenvalue problem, the critical buckling load of cracked nanobeams is obtained.

\section{Results and discussion}

This Section presents the influence of crack location, crack severity and material length scale parameter to thickness ratio on the nondimensional critical buckling load of simply supported cracked nanobeams. The nondimensional critical buckling load is defined as $\bar{P}=P_{c r} L^{2} / E I$. To validate the presented results, the critical buckling load of an intact microbram is compared with the obtained results by Mohammad-Abadi and Daneshmehr (2014). An excellent agreement is shown in Table 1. 
Table 1. Nondimensional critical buckling load $\bar{P}$ of intact microbeams $(E=1.44 \mathrm{GPa}, L=20 h$, $\ell=17.6 \mu \mathrm{m}, b=2 \mathrm{~h}, \nu=0.38)$

\begin{tabular}{|l|c|c|c|c|}
\hline & \multicolumn{4}{|c|}{$h[\mu \mathrm{m}]$} \\
\cline { 2 - 5 } & 17.6 & 52.8 & 88 & 123.2 \\
\hline \hline Ref. [25] & 60.3571 & 22.9608 & 19.9491 & 19.1188 \\
\hline Present paper & 60.4853 & 22.9962 & 19.9795 & 19.1479 \\
\hline
\end{tabular}

Ref. [25] - Mohammad-Abadi and Daneshmehr (2014)

To present a parametric study, a Timoshenko beam with $L=20 h, b=2 h$ and $\nu=0.38$ with simply supported boundary conditions and an open edge crack is considered. The effects of the flexibility constants $k_{1}, k_{2}, k_{3}$ and $k_{4}$ are shown in Tables 2-5. These tables present nondimensional critical buckling loads for different flexibility constants. As mentioned, this study introduces local flexibility by means of four constants which relate discontinuities of the cracked section with the axial force and bending moment. Therefore, different conditions of these four constants state different crack severities. It is found that the flexibility constant related to the bending moment $k_{1}$ is more effective on the buckling behavior of the cracked nanobeam than the other constants. In fact, the discontinuity in rotation due to bending has a great effect on the local flexibility introduced by the crack. However, the other constants $k_{2}, k_{3}$ and $k_{4}$ have a scant influence on the local flexibility, but this influence becomes larger when the crack severity is increased (Tables 3-5). Also, the crossover flexibility constants caused by the coupled effects between the axial force and bending moment $\left(k_{2}\right.$ and $\left.k_{4}\right)$ have similar influences on the critical buckling load (Tables 3 and 5).

Table 2. Effect of $k_{1}$ on the nondimensional critical buckling load $\bar{P}(\ell / h=1$ and $e=0.5)$

\begin{tabular}{|c|c|c|c|c|}
\hline$k_{1}$ & $k_{2}=k_{3}=k_{4}=0$ & $k_{2}=k_{3}=k_{4}=0.5$ & $k_{2}=k_{3}=k_{4}=1$ & $k_{2}=k_{3}=k_{4}=2$ \\
\hline \hline 0 & 60.4853 & 59.4491 & 58.4024 & 55.5903 \\
\hline 0.25 & 40.3538 & 39.7141 & 39.0139 & 37.1375 \\
\hline 0.5 & 29.0547 & 28.5334 & 28.0332 & 26.6845 \\
\hline 1 & 18.8317 & 18.1148 & 17.7901 & 16.9438 \\
\hline 2 & 10.7610 & 9.8532 & 9.6804 & 9.2153 \\
\hline
\end{tabular}

Table 3. Effect of $k_{2}$ on the nondimensional critical buckling load $\bar{P}(\ell / h=1$ and $e=0.5)$

\begin{tabular}{|c|c|c|c|c|}
\hline$k_{2}$ & $k_{1}=k_{3}=k_{4}=0$ & $k_{1}=k_{3}=k_{4}=0.5$ & $k_{1}=k_{3}=k_{4}=1$ & $k_{1}=k_{3}=k_{4}=2$ \\
\hline \hline 0 & 60.4853 & 28.3580 & 18.3580 & 9.9372 \\
\hline 0.25 & 60.4853 & 28.6881 & 18.2012 & 9.8551 \\
\hline 0.5 & 60.4853 & 28.5334 & 18.0786 & 9.7668 \\
\hline 1 & 60.4852 & 28.2029 & 17.7901 & 9.5905 \\
\hline 2 & 60.4852 & 27.5220 & 17.1818 & 9.2153 \\
\hline
\end{tabular}

Figure 3 shows the effect of the material length scale parameter to thickness ratio $\ell / h$ on the critical buckling load of Timoshenko cracked nanobeams. It is observed that the critical buckling load increases as $\ell / h$ increases, and this increase is decreased by the growing severity of the crack. Note that $\ell / h=0$ virtually indicates the results obtained from the classical continuum theory which neglects the couple stress effect.

The effects of crack location on the critical buckling load of simply supported nanobeams for different crack severities and $\ell / h$ ratios are respectively demonstrated in Figs. 4 and 5 . The critical buckling load is sensitive to crack location and is decreased when the crack gets near to 
Table 4. Effect of $k_{3}$ on the nondimensional critical buckling load $\bar{P}(\ell / h=1$ and $e=0.5)$

\begin{tabular}{|c|c|c|c|c|}
\hline$k_{3}$ & $k_{1}=k_{2}=k_{4}=0$ & $k_{1}=k_{2}=k_{4}=0.5$ & $k_{1}=k_{2}=k_{4}=1$ & $k_{1}=k_{2}=k_{4}=2$ \\
\hline \hline 0 & 60.4853 & 28.6977 & 18.4197 & 11.1871 \\
\hline 0.25 & 60.4853 & 28.6128 & 18.2461 & 10.5983 \\
\hline 0.5 & 60.4853 & 28.5334 & 18.0981 & 10.1732 \\
\hline 1 & 60.4852 & 28.4527 & 17.7901 & 9.6471 \\
\hline 2 & 60.4852 & 27.3721 & 17.5985 & 9.2153 \\
\hline
\end{tabular}

Table 5. Effect of $k_{4}$ on the nondimensional critical buckling load $\bar{P}(\ell / h=1$ and $e=0.5)$

\begin{tabular}{|c|c|c|c|c|}
\hline$k_{4}$ & $k_{1}=k_{2}=k_{3}=0$ & $k_{1}=k_{2}=k_{3}=0.5$ & $k_{1}=k_{2}=k_{3}=1$ & $k_{1}=k_{2}=k_{3}=2$ \\
\hline \hline 0 & 60.4853 & 28.8544 & 18.3579 & 9.9384 \\
\hline 0.25 & 60.4853 & 28.6879 & 18.2010 & 9.8552 \\
\hline 0.5 & 60.4853 & 28.5334 & 18.0787 & 9.7673 \\
\hline 1 & 60.4852 & 28.2045 & 17.7901 & 9.5911 \\
\hline 2 & 60.4851 & 27.5220 & 17.1817 & 9.2153 \\
\hline
\end{tabular}

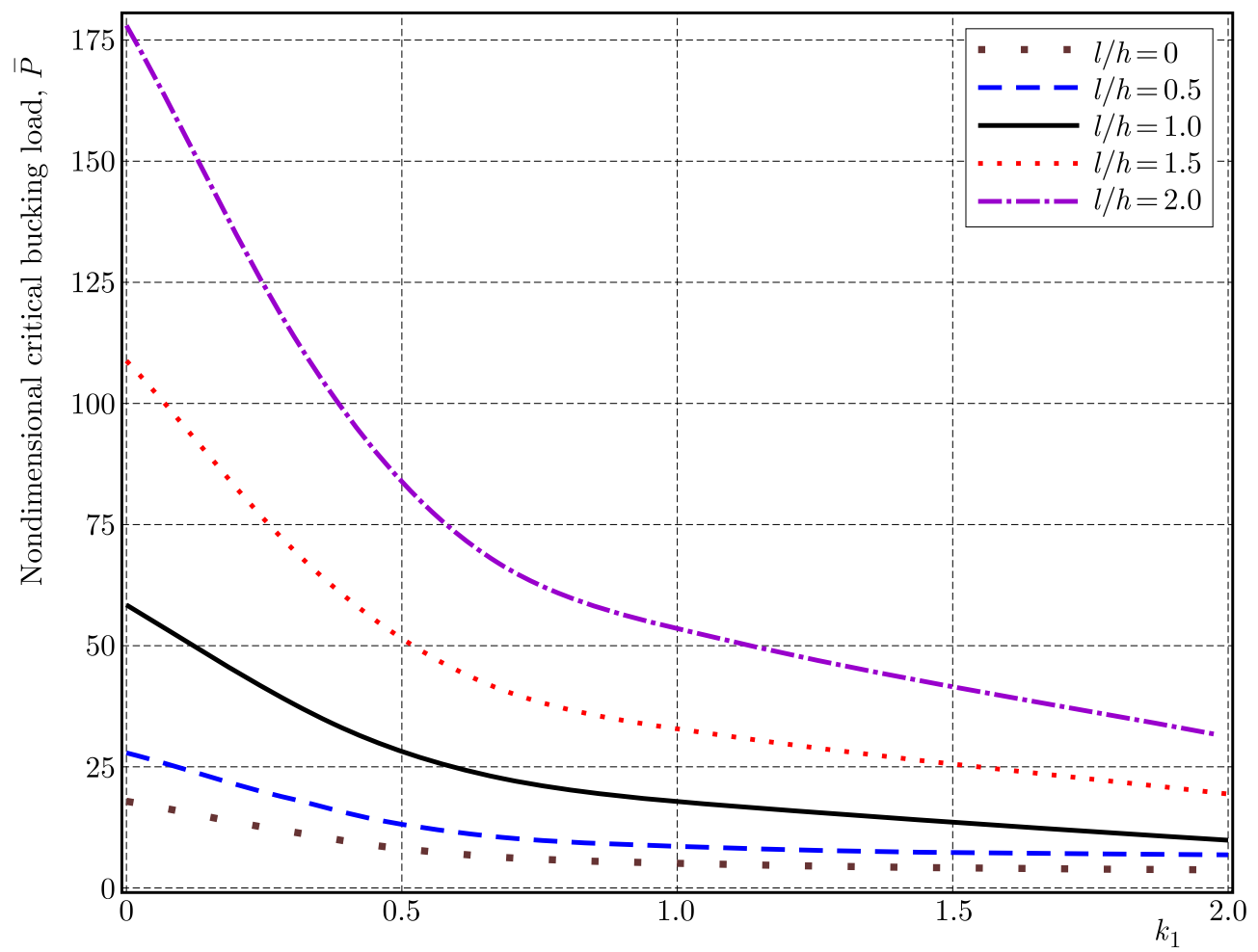

Fig. 3. Effects of $\ell / h$ ratio and crack severity on the nondimensional critical buckling load $\left(k_{2}=k_{3}=k_{4}=1\right.$ and $\left.e=0.5\right)$

beam midpoint. Also, the influence of the crack tends to be very small as the crack gets closer to the beam ends. By increasing the crack severity, the effect of crack location is increased; in other words, the crack severity is more effective when the crack is located at the midpoint of the beam $(e=0.5)$. Figure 5 indicates that the effect of crack location increases as the $\ell / h$ increases. Also, it is found that the $\ell / h$ ratio at different crack locations has different effects on the critical buckling load of cracked nanobeams, but these effects get similar when the crack gets near to the midpoint. 


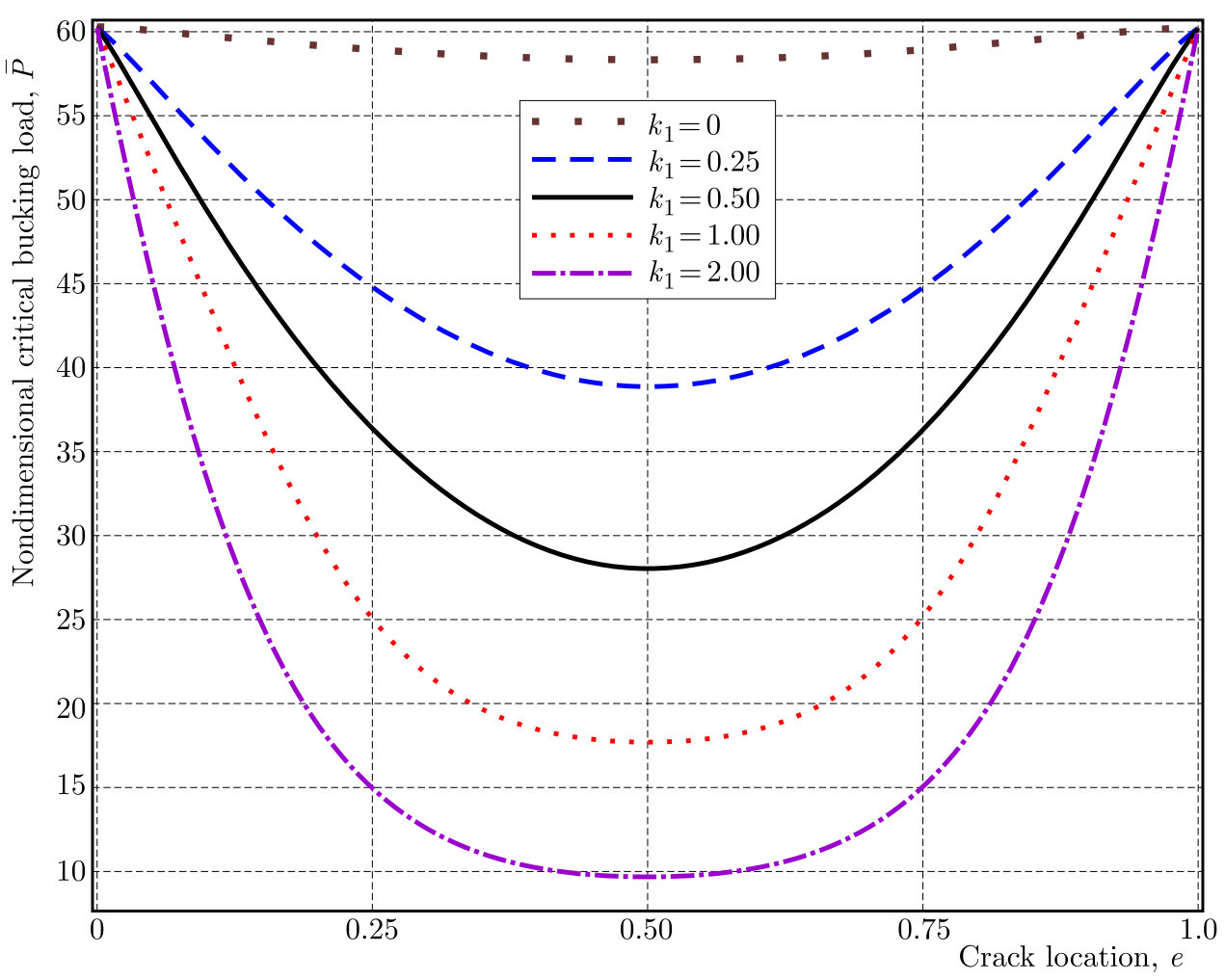

Fig. 4. Effects of crack location and crack severity on the nondimensional critical buckling load $\left(k_{2}=k_{3}=k_{4}=1\right.$ and $\left.\ell / h=1\right)$

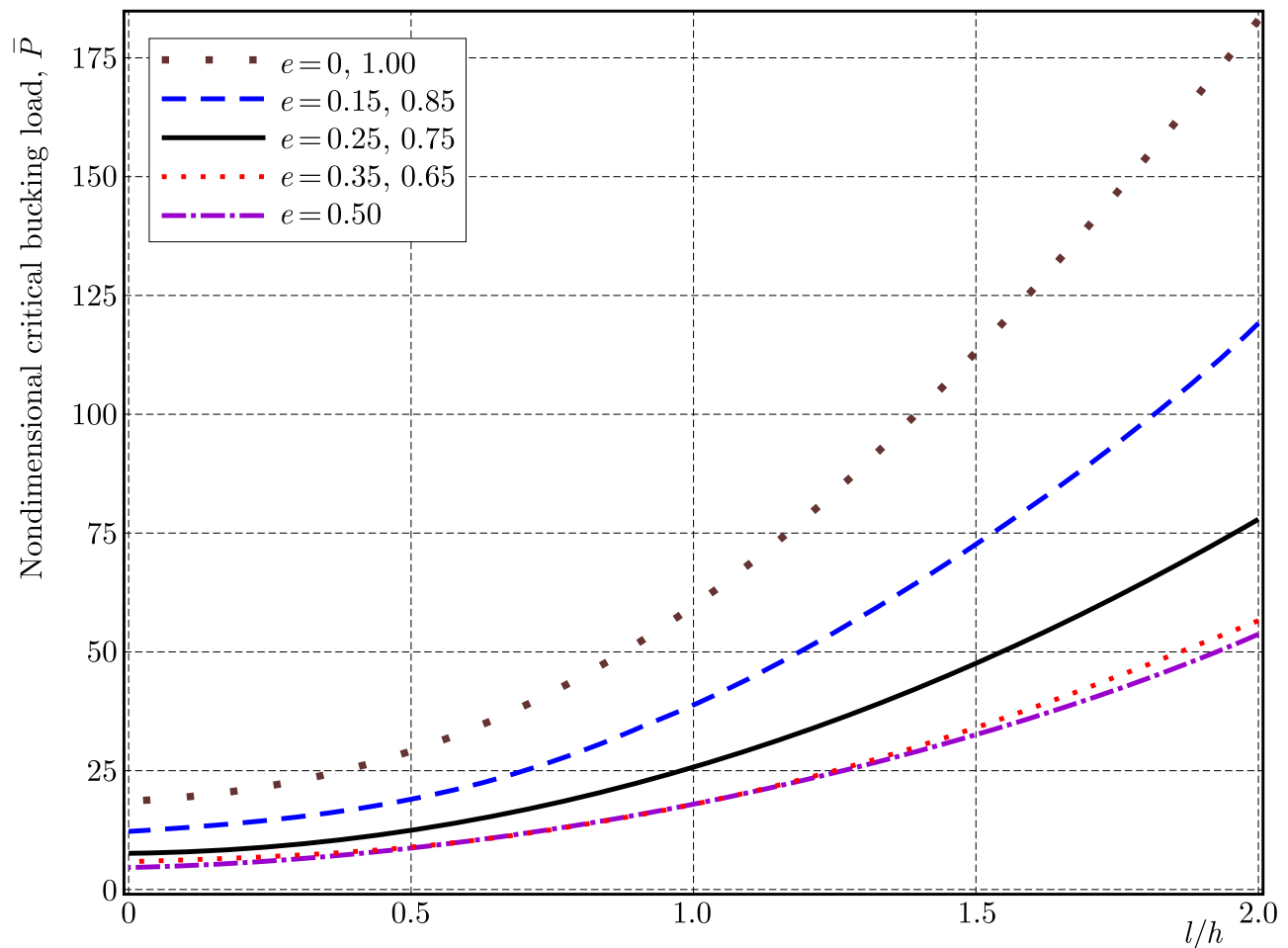

Fig. 5. Effects of crack location and $\ell / h$ ratio on the nondimensional critical buckling load $\left(k_{1}=k_{2}=k_{3}=k_{4}=1\right)$ 


\section{Conclusion}

The buckling behavior of cracked nanobeams is studied within the framework of Timoshenko beam theory anda modified couple stress theory. A material length scale parameter is used to capture the size effect. The crack is modeled as two massless elastic springs (rotational and longitudinal) which promote discontinuities at the cracked section. This study employs four flexibility constants which introduce crack severity by two discontinuities which are proportional to the bending moment and axial force transmitted through the cracked section. The principle of minimum potential energy is employed to derive the governing differential equations which are discretized using the GDQ method. The effects of crack severity, crack location and material length scale parameter to thickness ratio are studied.

The obtained results show that the first flexibility constant which connects the discontinuity in rotation with the bending moment makes the main part of the crack severity. The material length scale parameter to thickness ratio $\ell / h$ considerably affects the value of the critical buckling load which increases as the $\ell / h$ increases. The influence of $\ell / h$ is stronger at lower crack severities, in a general manner, reduction of the crack effects (crack severity and location) leads to a decrease in the influence of $\ell / h$. The critical buckling loads are significantly affected by crack location and are continuously decreased when the crack gets near to the midpoint of the nanobeam. Also, the crack location is more effective at high crack severities and $\ell / h$ ratios.

\section{References}

1. Adams R.D., Cawley P., Pye C.J., Stone B.J., 1978, A vibration technique for non-destructive assessing the integrity of structures, Journal of Mechanical Engineering Science, 20, 93-100

2. Akbarzadeh Khorshidi M., Shariati M., 2015, A modified couple stress theory for postbuckling analysis of Timoshenko and Reddy-Levinson single-walled carbon nanobeams, Journal of Solid Mechanics, 7, 4, 364-373

3. Akbarzadeh Khorshidi M., Shariati M., 2016a, An investigation of stress wave propagation in a shear deformable nanobeam based on modified couple stress theory, Waves in Random and Complex Media, 26, 243-258, DOI: 10.1080/17455030.2015.1137375

4. Akbarzadeh Khorshidi M., Shariati M., 2016b, Free vibration analysis of sigmoid functionally graded nanobeams based on a modified couple stress theory with general shear deformation theory, Journal of the Brazilian Society of Mechanical Sciences and Engineering, 38, 2607-261, DOI: $10.1007 / \mathrm{s} 40430-015-0388-3$

5. Asghari M., Kahrobaiyan M.H., Ahmadian M.T., 2010, A nonlinear Timoshenko beam formulation based on the modified couple stress theory, International Journal of Engineering Science, 48, 1749-1761, DOI: 10.1016/j.ijengsci.2010.09.025

6. Bellman R., Kashef B.G., Casti J., 1972, Differential quadrature: a technique for the rapid solution of nonlinear partial differential equations, Journal of Computer and Physics, 10, 40-52

7. Chati M., Rand R., Mukherjee S., 1007, Modal analysis of a cracked beam, Journal of Sound and Vibration, 207, 2, 249-270

8. Chaudhari T.D., Maiti S.K., 2000, A study of vibration of geometrically segmented beams with and without crack, International Journal of Solids and Structures, 37, 761-779

9. Dehrouyeh-Semnani A.M., Dehrouyeh M., Zafari-Koloukhi H., Ghamami M., 2015, Size-dependent frequency and stability characteristics of axially moving microbeams based on modified couple stress theory, International Journal of Engineering Science, 97, 98-112, DOI: 10.1016/j.ijengsci.2015.09.003

10. El Bikri K., Benamar R., Bennouna M.M., 2006, Geometrically non-linear free vibrations of clamped-clamped beams with an edge crack, Computers and Structures, 84, 485-502, DOI: 10.1016/j.compstruc.2005.09.030 
11. Freund L.B., Herrmann G., 1978, Dynamic fracture of a beam or plate in plane bending, Journal of Applied Mechanic, 76-APM-15, 112

12. Hasheminejad S.M., Gheshlaghi B., Mirzai Y., Abbasion S., 2011, Free transverse vibrations of cracked nanobeams with surface effects, Thin Solid Films, 519, 2477-2482, DOI: 10.1016/j.tsf.2010.12.143

13. Hosseini-Hashemi Sh., Fakher M., Nazemnezhad R., Sotoude Haghighi M.H., 2014, Dynamic behavior of thin and thick cracked nanobeams incorporating surface effects, Composites: Part B, 61, 66-72, DOI: 10.1016/j.compositesb.2014.01.031

14. Hsu J.Ch., Lee H.L., Chang W.J., 2011, Longitudinal vibration of cracked nanobeams using nonlocal elasticity theory, Current Applied Physics, 11, 1384-1388, DOI: 10.1016/j.cap.2011.04.026

15. Ke L.L., WAng Y.Sh., 2011, Size effect on dynamic stability of functionally graded microbeams based on a modified couple stress theory, Composite Structures, 93, 342-350, DOI: 10.1016/j.compstruct.2010.09.008

16. Ke L.L., Yang J., Kitipornchai S., 2009, Postbuckling analysis of edge cracked functionally graded Timoshenko beams under end shortening, Composite Structures, 90, 152-160, DOI: 10.1016/j.compstruct.2009.03.003

17. Kitipornchai S., Ke L.L., Yang J., Xiang Y., 2009, Nonlinear vibration of edge cracked functionally graded Timoshenko beams, Journal of Sound and Vibration, 324, 962-982, DOI: 10.1016/j.jsv.2009.02.023

18. Krawczuk M., Palacz M., Ostachowicz W., 2003, The dynamic analysis of cracked Timoshenko beams by spectral element method, Journal of Sound and Vibration, 264, 1139-1153

19. Lele S.P., MAiti S.K., 2002, Modelling of transverse vibration of short beams for crack detection and measurement of crack extension, Journal of Sound and Vibration, 257, 3, 559-583, DOI: $10.1006 /$ jsvi.5059

20. Loya J.A., Aranda-Ruiz J., Fernandez-Saez J., 2014, Torsion of cracked nanorods using a nonlocal elasticity model, Journal of Physics D: Applied Physics, 47, 115304, DOI: 10.1088/0022$-3727 / 47 / 11 / 115304$

21. Loya J., Lopez-Puente J., Zaera R., Fernandez-Saez J., 2009, Free transverse vibrations of cracked nanobeams using a nonlocal elasticity model, Journal of Applied Physics, 105, 044309, DOI: $10.1063 / 1.3068370$

22. Loya J.A., Rubio L., Fernandez-Saez J., 2006, Natural frequencies for bending vibrations of Timoshenko cracked beams, Journal of Sound and Vibration, 290, 640-653, DOI: 10.1016/j.jsv.2005.04.005

23. Ma H.M., Gao X.-L., Reddy J.N., 2008, A microstructure-dependent Timoshenko beam model based on a modified couple stress theory, Journal of the Mechanics and Physics of Solids, 56, 3379-3391, DOI: 10.1016/j.jmps.2008.09.007

24. Ma H.M., Gao X.-L., Reddy J.N., 2010, A nonclassical Reddy-Levinson beam model based on a modified couple stress theory, Journal for Multiscale Computational Engineering, 8, 2, 167-180

25. Mohammad-Abadi M., Daneshmehr A.R., 2014, Size dependent buckling analysis of microbeams based on modified couple stress theory with high order theories and general boundary conditions, International Journal of Engineering Science, 74, 1-14, DOI: 10.1016/j.ijengsci.2013.08.010

26. Mohammad-Abadi M., Daneshmehr A.R., 2015, Modified couple stress theory applied to dynamic analysis of composite laminated beams by considering different beam theories, International Journal of Engineering Science, 87, 83-102, DOI: 10.1016/j.ijengsci.2014.11.003

27. Okamura H., Watanabe K., Takano T., 1973, Applications of compliance concept in fracture mechanics, [In:] Progress in Flaw Growth and Fracture Toughness Testing, 536, J.G. Kaufman (Edit.), American Society for Testing and Materials, Philadelphia, 423-438

28. PARK S.K., Gao X.-L., 2006, Bernoulli-Euler beam model based on a modified couple stress theory, Journal Micromechanics and Microengineering, 16, 2355-2359 
29. PARK S.K., Gao X.-L., 2008, Variational formulation of a modified couple stress theory and its application to a simple shear problem, Zeitschrift für angewandte Mathematik und Physik, 59, 904-917

30. Rice J.R., 1972, The line spring model for surface flaws, [In:] The Surface Crack, Swedlow J.L. (Edit.), ASME, New York

31. Rice J.R., LEVy N., 1972, The part-through surface crack in an elastic plate, Journal of Applied Mechanics, 39, Trans. ASME, Series E, 94, 185-194

32. Shu C., 1991, Generalized differential-integral quadrature and application to the simulation of incompressible viscous flows including parallel computation, Ph.D. Thesis, University of Glasgow

33. Shu C., Du H., 1997, Implement of clamped and simply supported boundary conditions in the GDQ free vibration analysis of beams and plates, International Journal of Solids and Structures, 19, 59-68

34. Simsek M., 2010, Dynamic analysis of an embedded microbeam carrying a moving microparticle based on the modified couple stress theory, International Journal of Engineering Science, 48, 1721-1732, DOI: 10.1016/j.ijengsci.2010.09.027

35. ThARP T., 1987, A finite element for edge-cracked beam columns, International Journal for Numerical Methods in Engineering, 24, 1941-1950

36. Torabi K., Nafar Dastgerdi J., 2012, An analytical method for free vibration analysis of Timoshenko beam theory applied to cracked nanobeams using a nonlocal elasticity model, Thin Solid Films, 520, 6595-6602, DOI: 10.1016/j.tsf.2012.06.063

37. Xia W., Wang L., Yin L., 2010, Nonlinear non-classical microscale beams: Static bending, postbuckling and free vibration, International Journal of Engineering Science, 48, 12, 2044-2053, DOI: 10.1016/j.ijengsci.2010.04.010

38. Yan T., Kitipornchai S., Yang J., 2011, Parametric instability of functionally graded beams with an open edge crack under axial pulsating excitation, Composite Structures, 93, 1801-1808, DOI: $10.1016 /$ j.compstruct.2011.01.019

39. Yang F., Chong A.M., Lam D.C.C., Tong P., 2012, Couple stress based strain gradient theory of elasticity, International Journal of Solids and Structures, 39, 2731-2743, DOI: 10.1016/S0020$-7683(02) 00152-\mathrm{X}$

40. YAng J., Chen Y., 2008, Free vibration and buckling analyses of functionally graded beams with edge cracks, Composite Structures, 83, 48-60, DOI: 10.1016/j.compstruct.2007.03.006 